\title{
Erratum: Nonequilibrium polarization dynamics in antiferroelectrics [Phys. Rev. B 96, 014104 (2017)]
}

\author{
M. M. Vopson and X. Tan
}

(Received 7 May 2019; published 15 May 2019)

DOI: 10.1103/PhysRevB.99.209902

While working on a new article [1] on the same sample and using some of the existing data, we recently detected an error in our previously published paper. The main error is in the Experiments and Results section on p. 014104 of the original paper. From the double hysteresis loop at room temperature (black loop in Fig. 1 here measured at $22^{\circ} \mathrm{C}$ ), we extracted for numerical calculations the following parameters of the $\mathrm{Pb}_{0.99} \mathrm{Nb}_{0.02}\left[\left(\mathrm{Zr}_{0.57} \mathrm{Sn}_{0.43}\right)_{1-y} \mathrm{Ti}_{y}\right]_{0.98} \mathrm{O}_{3}(\mathrm{PNZST} 43 / 6 / 2)$ antiferroelectric sample: $2 P_{s}=37.4, P_{A}=\left|P_{B}\right|=P_{s}=18.7 \mu \mathrm{C} / \mathrm{cm}^{2}, \pm E_{0}=16.6$, and $\pm E_{s}=36 \mathrm{kV} / \mathrm{cm}$. These are not all correct. The $2 P_{s}=$ $37.4-\mu \mathrm{C} / \mathrm{cm}^{2}$ value appears to be double the real value as seen in Fig. 1 here (Fig. 3 in the original paper). Consequently, the correct values are $2 P_{s}=18.7$ and $P_{A}=\left|P_{B}\right|=P_{S}=9.35 \mu \mathrm{C} / \mathrm{cm}^{2}$. We believe this tiny unintentional error was due to the confusion around the vertical axis that is usually reported as polarization, whereas in our paper is in fact $2 \times$ polarization of a single sublattice (see Fig. 1 in our paper). The theoretical section of the paper as well as all other sections remain fully unchanged, and this detected error only changes marginally some of the numerical calculations. Using the new $2 P_{s}=18.7 \mu \mathrm{C} / \mathrm{cm}^{2}$ value, we determined the activation energy of the domain nucleation process for this particular antiferroelectric sample to be $W_{b}=1.05$ instead of $W_{b}=1.07 \mathrm{eV}$ as previously reported. A slightly more significant variation is in the critical volume of the polar nucleus, which is now $V^{*}=190 \times 10^{-27}$ instead of $V^{*}=98 \times 10^{-27} \mathrm{~m}^{3}$. This corresponds to a linear length scale of 3.57 instead of $2.86 \mathrm{~nm}$ as previously reported, which again is only a marginal change. However, these numbers were wrongly reported in the Abstract, Experiments and Results, and Conclusions sections of the article in the original paper.

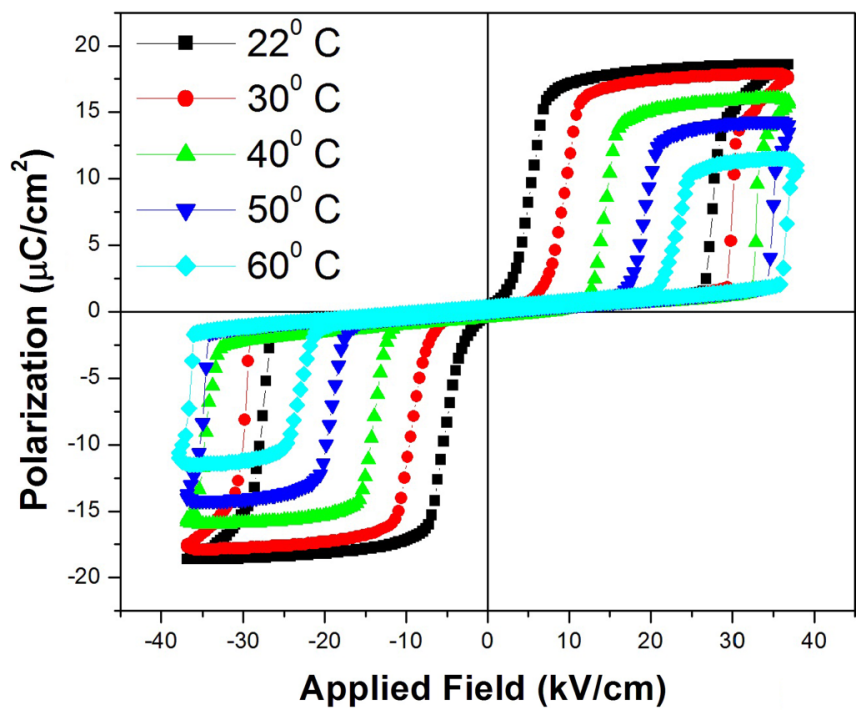

FIG. 1. Polarization hysteresis loops as a function of temperature for PNZST 43/6/2 antiferroelectric sample.

[1] M. Vopson, X. Tan, X. E. Namvar, M. Belusky, S. Thompson, V. Kuncser, F. Plazaola, I. Unzueta, and C. Tang, Sub-lattice polarization states in anti-ferroelectrics and their relaxation process, Current Appl. Phys. 19, 651 (2019). 